\title{
Die Biegefestigkeit von Keilzinkenverbindungen aus Brettern der Buche (Fagus silvatica $\mathbf{L}$.)
}

\author{
M. Frese • H. J. Blaß
}

Published online: 19 September 2006

(C) Springer-Verlag 2006

Zusammenfassung Die Biegefestigkeit von Brettschichtholz aus Buche hängt ebenso wie beim Nadelholz von der Festigkeit der Bretter und der Keilzinkenverbindungen ab. Über den Einfluss einer visuellen oder maschinellen Sortierung von Buchenbrettern auf deren Zugfestigkeit und damit auf die Biegefestigkeit von Brettschichtholz wurde in einer kürzlich abgeschlossenen Forschungsarbeit berichtet. Darin wurde gezeigt, dass in Abhängigkeit von einer zunächst variablen Keilzinkenbiegefestigkeit eine visuelle Sortierung eine charakteristische Biegefestigkeit des Brettschichtholzes von $36 \mathrm{~N} / \mathrm{mm}^{2}$ und eine maschinelle Sortierung von $48 \mathrm{~N} / \mathrm{mm}^{2}$ ermöglicht. Der Nachweis, ob die Sortierungen auch die dafür erforderliche Keilzinkenbiegefestigkeit gewährleisten, stand noch aus. In der vorliegenden Arbeit wird nun über die unterschiedliche Wirkung von visuellen oder maschinellen Sortierungen auf die Biegefestigkeit von Keilzinkenverbindungen berichtet. Anhand von 367 Biegeversuchen an Keilzinkenverbindungen aus Buche wird gezeigt, dass eine Sortierung von Buchenbrettern in die Sortierklassen LS10 oder LS13 nach DIN 4074-5 bezüglich der Keilzinkenbiegefestigkeit die notwendigen Vorraussetzungen für die Herstellung von GL36 aus Buche schafft. Eine geeignete maschinelle Sortierung nach dem dynamischen Elastizitätsmodul ermöglicht GL48.

M. Frese ( $)$ H. J. Blaß

Lehrstuhl für Ingenieurholzbau und Baukonstruktionen,

Universität Karlsruhe,

Kaiserstraße 12,

76131 Karlsruhe, Germany

e-mail: matthias.frese@holz.uka.de

\section{The bending strength of finger joints manufactured} from beech wood (Fagus silvatica $\mathrm{L}$.)

\begin{abstract}
The bending strength of glulam depends on both board strength and finger joint strength. In the case of softwood glulam this fact is well known from experience. It was recently reported by the authors that visual strength grading of beech provides a characteristic glulam bending strength of $36 \mathrm{~N} / \mathrm{mm}^{2}$ and mechanical strength grading of $48 \mathrm{~N} / \mathrm{mm}^{2}$. Therefore, adequate values for the characteristic finger joint bending strength have to be met. At the time those values were unknown. It was the aim of the following project to study the influence of visual or mechanical strength grading techniques on the finger joint bending strength. Bending tests on finger joints were carried out. The specimens were manufactured from visually strength graded boards according to the German standard DIN 4074-5 and from mechanically strength graded boards according to the dynamic MOE. The test results confirm that visual strength grading provides strength class GL36 and mechanical grading provides GL48.
\end{abstract}

\section{Einleitung und Hintergrund}

In dem Forschungsvorhaben ,Biegefestigkeit von Brettschichtholz aus Buche“ zeigten Blaß et al. (2004) den unterschiedlichen Einfluss von visuellen und maschinellen Sortierverfahren für Buchenbretter auf die Biegefestigkeit von kombiniert aufgebauten Brettschichtholzträgern auf: An etwa 1900 Buchenbrettern wurden dazu als festigkeitsund steifigkeitsbestimmende Parameter die Ästigkeit nach DIN 4074 Teil 5, die Rohdichte und der dynamische Elastizitätsmodul aus Längsschwingungen bestimmt. An die Häufigkeitsverteilungen dieser Daten wurden theoretische 
Tabelle 1 Sortiermodelle für Buchenbretter

Table 1 Grades of beech

\begin{tabular}{llcc}
\hline Modell & Ästigkeit & $\begin{array}{c}\text { Elastizitätsmodul } \\
{\left[\mathrm{N} / \mathrm{mm}^{2}\right]}\end{array}$ & $\begin{array}{c}\text { Charakteristische } \\
\text { Zugfestigkeit }\left[\mathrm{N} / \mathrm{mm}^{2}\right]\end{array}$ \\
\hline LS10 & $D E B \leq 0,33$ & - & 22 \\
LS13a & $D E B \leq 0,20$ & - & 27 \\
LS13b & $D E B \leq 0,042$ & - & 31 \\
MSa & $D E B \leq 0,20$ & $15000<E_{\text {dyn }}$ & 40 \\
MSb & $D E B \leq 0,042$ & $15000<E_{\text {dyn }}$ & 48 \\
\hline
\end{tabular}

$D E B=$ Ästigkeit für den Einzelast nach DIN 4074 Teil 5

$E_{\mathrm{dyn}}=$ dynamischer Elastizitätsmodul ermittelt aus

Längsschwingungen

Verteilungsfunktionen angepasst. Diese fanden in einem neu entwickelten Rechenmodell Verwendung, mit dem auf der Grundlage einer Regressionsrechnung die mechanischen Eigenschaften von beliebig sortierten Buchenbrettern wirklichkeitsnah simuliert werden können. Mit dem Rechenmodell lässt sich dann die Zugfestigkeit der Bretter ermitteln sowie die Biegefestigkeit daraus aufgebauter Brettschichtholzträger mithilfe der Finite-Elemente-Methode berechnen. Die Ergebnisse zahlreicher Trägerversuche nach DIN EN 408 in Bauteilgröße bestätigen eine zuverlässige Tragfähigkeitsvorhersage des Rechenmodells.

Daraufhin wurden mithilfe des Rechenmodells fünf praxisrelevante Sortiermodelle für Buchenbretter entwickelt, die in erster Linie eine unterschiedliche Auswirkung auf die charakteristische Zugfestigkeit der sortierten Bretter besitzen. In Tabelle 1 sind für jedes Sortiermodell die Sortierkriterien, deren zugehörige Grenzwerte und die cha- rakteristische Zugfestigkeit aufgeführt. Anhand des Rechenmodells wurde für die fünf Sortiermodelle jeweils ein funktionaler Zusammenhang zwischen der charakteristischen Biegefestigkeit der Träger und der charakteristischen Biegefestigkeit von Keilzinkenverbindungen ermittelt (Abb. 1). Dabei war es notwendig, die Keilzinkenbiegefestigkeit zunächst als veränderliche Größe vorzugeben. Zum einen lagen über die Keilzinkenfestigkeit aus visuell oder maschinell sortierten Buchenbrettern bislang nur unzureichende Kenntnisse vor. Zum anderen ist die Keilzinkenfestigkeit produktionsbedingt grundsätzlich als schwankender Wert zu betrachten. In Abb. 1 ist für jedes Sortiermodell die Einstufung in Brettschichtholz-Festigkeitsklassen bis GL48 in Abhängigkeit von einer dafür erforderlichen charakteristischen Biegefestigkeit der Keilzinken dargestellt. Demnach ist für eine Festigkeitsklasse GL48 die maschinelle Sortierung MSb und eine charakteristische Keilzinkenbiegefestigkeit von $70 \mathrm{~N} / \mathrm{mm}^{2}$ erforderlich. Um die Festigkeitsklasse GL36 mit der visuellen Sortierung LS13b zu erreichen, ist eine charakteristische Keilzinkenbiegefestigkeit von mindestens $56 \mathrm{~N} / \mathrm{mm}^{2}$ Voraussetzung. Um zu überprüfen, ob diese Anforderungen an die Keilzinkenbiegefestigkeit tatsächlich erfüllt werden können, wurden im Anschluss an das Forschungsvorhaben die Auswirkungen einer visuellen und maschinellen Sortierung von Buchenbrettern auf die Biegefestigkeit von Keilzinken weiter untersucht. Die Ergebnisse werden in dieser Veröffentlichung dargestellt und sollen einen weiteren Beitrag für die Bemessung von Brettschichtholz aus Buche leisten.
Abb. 1 Charakteristische Brettschichtholz-Biegefestigkeit in Abhängigkeit vom Sortiermodell und der charakteristischen Keilzinkenbiegefestigkeit; Zuordnung zu Festigkeitsklassen für eine Trägerhöhe von $600 \mathrm{~mm}$ Fig. 1 Characteristic glulam bending strength depending on the grades and the characteristic finger joint bending strength; glulam strength classes referring to $600 \mathrm{~mm}$ deep beams

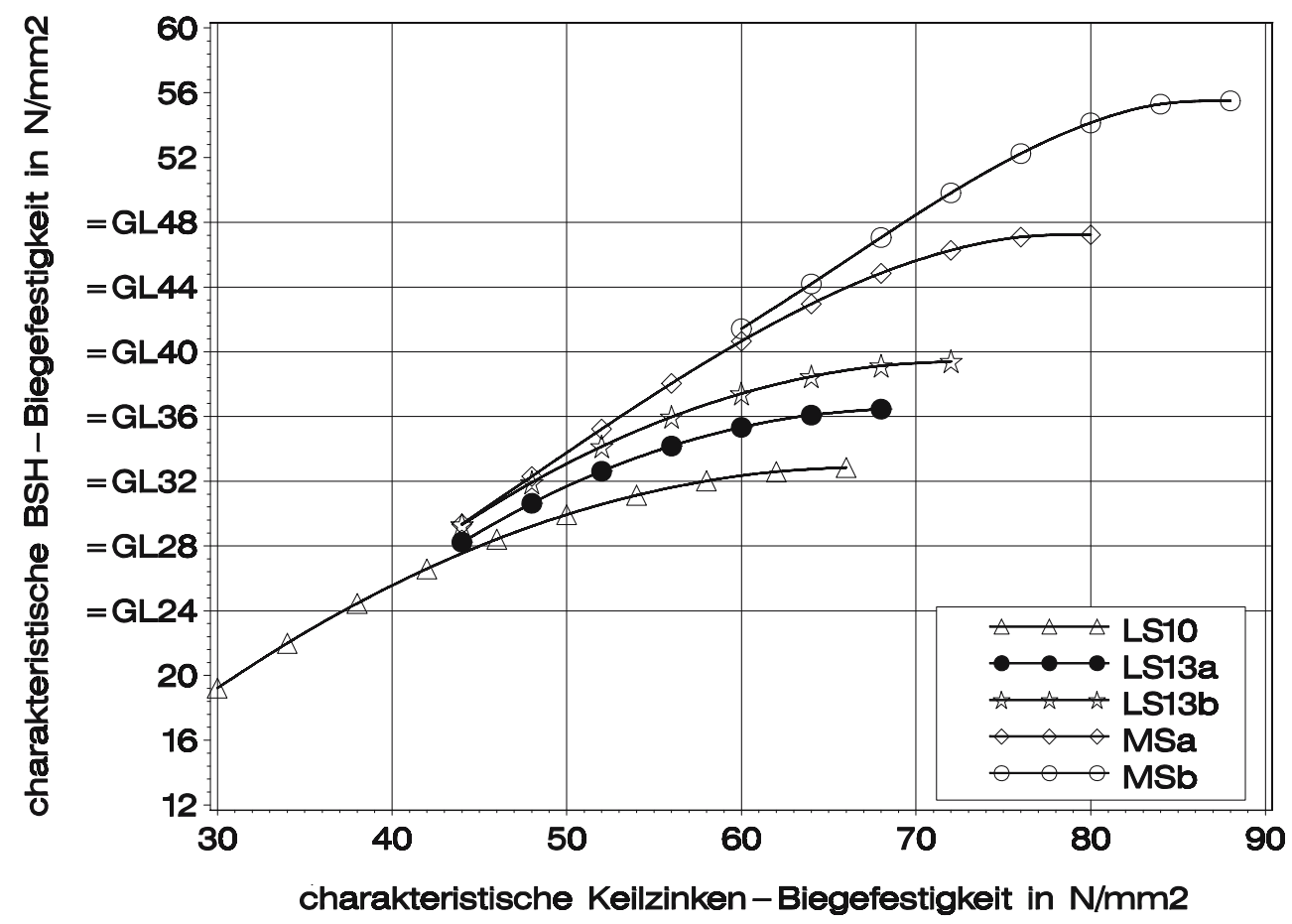




\section{Material und Methoden}

\subsection{Allgemeines}

Für das zuvor genannte Forschungsvorhaben wurden zum einen Keilzinkenproben aus visuell sortierten Buchenbrettern und zum anderen Versuchsträger aus maschinell sortierten Brettern produziert. Die visuelle Sortierung entsprach den Sortierklassen LS10 und LS13. Die maschinelle Sortierung der Bretter für die Versuchsträger erfolgte nach dem aus Längsschwingungen ermittelten dynamischen Elastizitätsmodul, wobei die Bretter nach dem System in Tabelle 2 klassifiziert wurden. Alle Keilzinkenverbindungen aus maschinell sortierten Brettern wurden im Anschluss an die Biegeprüfung der Versuchsträger aus unbeschädigten Trägerbereichen sorgfältig ausgesägt. Anstoß zu dieser Gewinnung von Keilzinkenproben gab die Arbeit von Ehlbeck et al. (1984). Darin wurde an einem kleinen Stichprobenumfang gezeigt, dass es technisch möglich ist, Keilzinkenproben aus geprüften Trägern herzustellen. Eine eindeutig dokumentierte Zuordnung der Brett-Klasse gemäß Tabelle 2 zu den Lamellen im Versuchsträger ermöglichte es, Stichproben für Keilzinken-Biegeprüfkörper zu entnehmen, die sich bezüglich der Klasse der miteinander verbundenen Bretter voneinander unterschieden. Keilzinken aus Brettern der Klasse 1 blieben unberücksichtigt, weil eine maschinelle Sortierung in diese Klasse für Lamellen nicht praxisrelevant ist. Alle Keilzinkenverbindungen wurden von einem Brettschichtholzhersteller mit dem für Nadelholz gebräuchlichen Profil 15/3,8 gefertigt. Als Kleber wurde Kauramin ${ }^{\circledR}$-Leim 681 flüssig und Kauramin ${ }^{\circledR}$-Härter 686 flüssig verwendet. Dieser ist für das Verkleben tragender Bauteile im Sinne von DIN 1052 geeignet, wobei darin die Verwendung von Laubholz für Brettschichtholz nicht geregelt wird.

\subsection{Material}

Die Buchenbretter zur Herstellung der Keilzinkenproben und Versuchsträger wurden von drei in Deutschland ansässigen Sägewerken geliefert. Das Material kam jeweils zu einem Drittel aus dem Spessart (Bayern), aus Nordhessen und aus dem Schönbuch (Baden-Württemberg). Es handelte sich um unbesäumte Bretter, die in den Sägewerken

Tabelle 2 Klassifizierung nach dem dynamischen

Elastizitätsmodul

Table 2 Grading scheme according to the dynamic MOE

\begin{tabular}{lc}
\hline $\begin{array}{l}\text { Brett- } \\
\text { Klasse }\end{array}$ & $\begin{array}{c}\text { Grenzen für den } \\
\text { dynamischen } \\
\text { Elastizitätsmodul } \\
{\left[\mathrm{N} / \mathrm{mm}^{2}\right]}\end{array}$ \\
\hline 1 & $\begin{array}{c}E_{\text {dyn }} \leq 13000 \\
2\end{array}$ \\
3 & $13000<E_{\text {dyn }} \leq 14000$ \\
4 & $15000<E_{\text {dyn }} \leq 15000$ \\
5 & $16000<E_{\text {dyn }} \leq 16000$ \\
\hline
\end{tabular}

bereits $1 \frac{1}{2}$ bis $2 \frac{1}{2}$ Jahre an der freien Luft lagerten. Aufgrund von Ästen und Rotkern wurde das Material in die Güteklassen B und C gemäß Verordnung über gesetzliche Handelsklassen für Rohholz eingestuft. Die Lagerware war ursprünglich für die Herstellung von Treppen, Leimholzplatten und Polstermöbelgestellen vorgesehen. Die unbesäumten Bretter wurden in zwei Sägewerken zuerst technisch getrocknet und dann parallel besäumt. In dem dritten Werk wurden sie zuerst besäumt und anschließend getrocknet. Die technische Trocknung auf 12\% Holzfeuchte nahm 10 bis 14 Tage in Anspruch. Im Labor wurden Bretter mit Markröhre und groben Fehlern aussortiert. Das verbleibende Material wurde dann für die Herstellung der Keilzinkenproben, die visuell sortiertes Material repräsentieren sollten, und der Versuchsträger vorbereitet. Anforderungen an das Holz im Bereich der Zinkung wurden der DIN EN 385 entnommen. An drei Produktionsterminen im Dezember 2003 und Februar sowie März 2004, an denen jeweils nur Holz einer Herkunft verarbeitet wurde, wurden die Keilzinkenproben aus visuell sortiertem Material und die Versuchsträger aus klassifizierten Brettern hergestellt.

Eine Sortierung in die Klassen LS10 und LS13 nach DIN 4074 Teil 5 besitzt erwartungsgemäß keinen Einfluss auf die Biegefestigkeit der Keilzinken: Bezüglich der Markröhre, Jahrringbreite und Faserneigung wird zwischen LS10 und LS13 im Falle von Buche nicht unterschieden. Für das Brettende in der Nähe der Keilzinkung gelten für LS10 und LS13 die gleichen Kriterien nach DIN EN 385. Blaß et al. (2004) konnten zeigen, dass der Mittelwert bzw. die Standardabweichung der Rohdichte bei $12 \%$ Holzfeuchte für LS10 und LS13 jeweils $675 \mathrm{~kg} / \mathrm{m}^{3}$ bzw. $35 \mathrm{~kg} / \mathrm{m}^{3}$ beträgt. Diese Werte wurden für LS10 an 1523 und für LS13 an 1202 Brettern ermittelt. Die strukturellen Eigenschaften des Holzes im Bereich der Keilzinkung sind folglich für beide Sortierklassen gleich. Insofern werden an den drei Stichproben in Tabelle 3 ohne Unterscheidung nach der Sor-

Tabelle 3 Stichproben der Keilzinkenverbindungen Table 3 Samples of finger joint specimens

\begin{tabular}{lcccc}
\hline Holzherkunft & Spessart & Nordhessen & Schönbuch & $\Sigma$ \\
\hline \multicolumn{4}{c}{ 3 Stichproben, die visuell sortiertes Material repräsentieren } \\
Anzahl & 31 & 56 & 21 & 108 \\
Breite/Dicke [mm] & $110 / 34$ & $100 / 30$ & $105 / 36$ & \\
\multicolumn{1}{l}{ 12 Stichproben, die } & maschinell & sortiertes & Material repräsentieren \\
Klasse 2 & 21 & 20 & 22 & 63 \\
Klasse 3 & 25 & 22 & 22 & 69 \\
Klasse 4 & 18 & 22 & 22 & 62 \\
Klasse 5 & 24 & 19 & 22 & 65 \\
Anzahl & 88 & 83 & 88 & 259 \\
Breite/Dicke [mm] & $110 / 33$ & $100 / 29$ & $105 / 34$ & \\
\hline
\end{tabular}




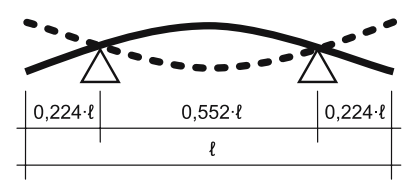

Abb. 2 Biegeschwingung I. Ordnung in Dickenrichtung; die Lage der Keilzinkenverbindung ist in Stabmitte.

Fig. 2 Flat wise flexural mode; the position of the finger joint is in the middle of the specimen

Tabelle 4 Statistische Kennwerte der Rohdichte bei 12\% Holzfeuchte in $\mathrm{kg} / \mathrm{m}^{3}$

Table 4 Apparent density statistics in $\mathrm{kg} / \mathrm{m}^{3}$ at $12 \%$ moisture content

\begin{tabular}{lccccc}
\hline Brettmaterial & $n$ & $\bar{x}$ & $s$ & $\min$ & $\max$ \\
\hline für Keilzinken & 263 & 689 & 41,4 & 574 & 802 \\
für Versuchsträger & 1888 & 676 & 34,7 & 575 & 818 \\
\hline
\end{tabular}

tierklasse die Festigkeitseigenschaften von Keilzinken aus visuell sortiertem Material bestimmt. Da für das Brettende der maschinell sortierten Bretter ebenso die Bedingungen nach DIN EN 385 eingehalten wurden, sind die 12 Stichproben in Tabelle 3 geeignet, den Einfluss einer maschinellen Sortierung auf die Keilzinkenbiegefestigkeit zu bestimmen.

In Tabelle 4 sind die statistischen Kennwerte der Rohdichte bei $12 \%$ Holzfeuchte für das Brettmaterial zur Herstellung der Keilzinkenproben und Versuchsträger zusammengestellt.

\subsection{Versuchsdurchführung}

Als Bezugsgröße zur Keilzinkenbiegefestigkeit wurde vor der Festigkeitsprüfung an allen Proben der dynamische Biege-Elastizitätsmodul anhand einer Biegeschwingung gemäß Abb. 2 bestimmt. Diese Methode wurde von Görlacher (1984) für Anwendungen im Holzbau weiterentwickelt und ist demnach für die zerstörungsfreie und zuverlässige Ermittlung des Elastizitätsmoduls geeignet. Die von ihm angegebene Gleichung wurde ohne den Term zur Berücksichtigung des Schubeinflusses verwendet. Das führt auf Gl. 1. Darin bedeuten: $E=$ Elastizitätsmodul, $\ell=$ Probenlänge, $f_{0}=$ Frequenz, $\rho=$ Rohdichte, $m_{n}=$ Konstante, die von der Ordnung der Schwingung abhängt, hier 4,73 und $i=$ Trägheitsradius in Richtung der Dicke.

Die Flachkant-Biegeprüfung erfolgte nach DIN EN 408 mit einer Stützweite von $15 \cdot d$. Keilzinkenproben aus visuell sortierten Brettern wurden unmittelbar nach der Herstellung geprüft. Solche aus maschinell sortierten Brettern ein Jahr nach der Herstellung der Keilzinkenverbindungen. Diese Verzögerung war bedingt durch die Herstellung der Keilzinkenproben aus geprüften Versuchsträgern. Nach der Festigkeitsprüfung wurden die Holzfeuchte und die Rohdichte an den verbleibenden
Bruchstücken nach DIN EN 408 ermittelt.

$$
E=\frac{4 \cdot \pi^{2} \cdot \ell^{4} \cdot f_{0}^{2} \cdot \rho}{m_{n}^{4} \cdot i^{2}}
$$

\section{Versuchsergebnisse}

\subsection{Keilzinkenqualität}

Die Proben aus visuell sortierten Brettern wurden in vier Qualitäten eingeteilt. Dabei wird zunächst zwischen Verbindungen mit produktionsbedingten Eigenschaften und nicht ordnungsgemäßen Proben unterschieden. Produktionsbedingte Eigenschaften von Keilzinken werden durch veränderliche Voraussetzungen bei der Herstellung verursacht (z.B. abnehmende Schärfe der Fräser während der Standzeit, verbleibende offene Zeit des Klebers, ungleichförmiger Klebstoffauftrag, gegenseitige Verdrehung der Brettenden vor dem Verpressen, schwankender Pressdruck, über den Querschnitt ungleichförmig verteilter Pressdruck). Je nach Zustand der Verbindung erhalten diese die Bewertungen ,sehr gut“, ,gut“ und ,,mit Zinkensprung“. Nicht ordnungsgemäße Verbindungen besitzen unzulässige Eigenschaften im Sinne von DIN EN 385. Solche Verbindungen sind in der Regel im Brettschichtholz nicht vorhanden, weil sie durch eine ordnungsgemäße Vorbereitung der Brettenden im Vorfeld ausgeschlossen werden. Sie erhalten die Bewertung ,nicht ordnungsgemäß““.

Die entsprechenden Kriterien für die vier Bewertungen sind in Tabelle 5 aufgeführt. Bei den Proben aus maschinell sortierten Brettern wurde keine weitere Unterscheidung bezüglich der produktionsbedingten Eigenschaften vorgenommen, weil der Zusammenhang zwischen Festigkeit und Qualität einer Keilzinkenverbindung anhand der Proben aus visuell sortierten Brettern später hinreichend deutlich wird. Die Auswertung in den Abschn. 3.2 und 3.3 erfolgt für Keilzinkenproben mit den Bewertungen ,sehr gut“, „gut“ und „mit Zinkensprung“, weil diese unter normalen praktischen Bedingungen produziert werden und nach der Schichtverklebung der Lamellen wirklich im Brettschicht-

Tabelle 5 Qualitäten

Table 5 Finger joint qualities

\begin{tabular}{ll}
\hline Bewertung & Kriterien \\
\hline sehr gut & $\begin{array}{l}\text { Fräsbild und Verklebung einwandfrei } \\
\text { (Abb. 3a) }\end{array}$ \\
gut & $\begin{array}{l}\text { Fräsbild einwandfrei, Verklebung } \\
\text { unzureichend (Abb. 3b) }\end{array}$ \\
mit Zinkensprung & $\begin{array}{l}\text { Zinken greifen unregelmäßig ineinander } \\
\text { (Abb. 3c) }\end{array}$ \\
nicht ordnungsgemäß & $\begin{array}{l}\text { Äste, Risse, festigkeitsmindernde Fäule } \\
\text { oder ähnliches befinden sich im } \\
\text { unzulässigen Abstand zum Zinkengrund }\end{array}$ \\
&
\end{tabular}


Abb. 3 Beispielhafte Bilder für Keilzinkenqualitäten: sehr gut: vorwiegendes Holzversagen im Zinkengrund (a), gut: Herausziehen der Zinken in der Biegezugzone (b) und mit Zinkensprung: Ansicht der Biegedruckzone (c)

Fig. 3 Pictures exemplifying the finger joint quality: very good: wood failure (a), good: failure of the glue line (b) and poor fitting (c)

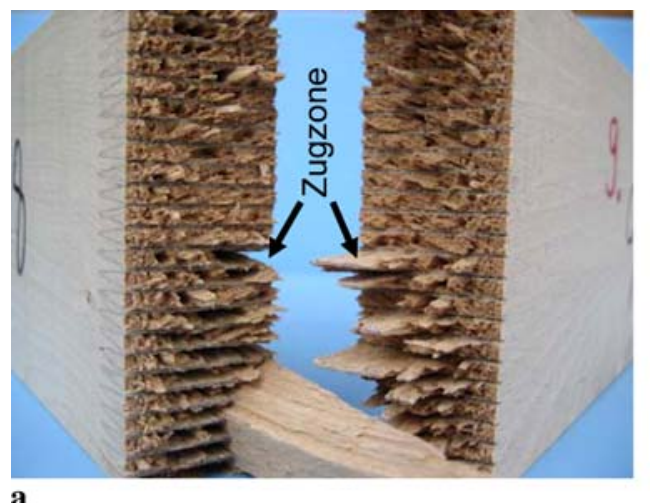

a

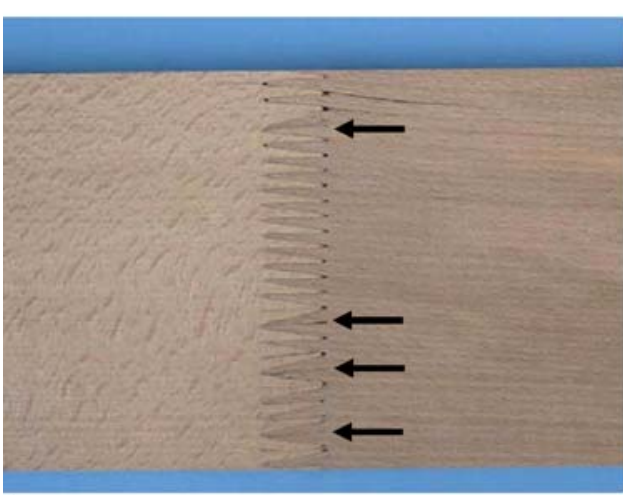

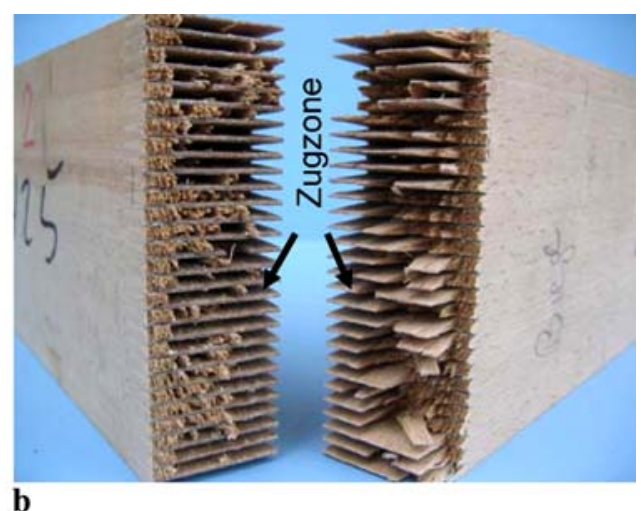

b holzträger vorkommen. Insgesamt standen damit die 108 „,visuellen“ und 259 „,maschinellen“ Proben aus Tabelle 3 zur Verfügung. Im Abschn. 3.4 werden dann ergänzende Hinweise zu den Festigkeitswerten von 39 nicht ordnungsgemäßen Proben gegeben.

\subsection{Holzfeuchte und Rohdichte ermittelt nach DIN EN 408}

An den Prüfkörpern wurden an jedem Brettende die Holzfeuchte und die Rohdichte nach DIN EN 408 bestimmt.

Tabelle 6 Statistische Kennwerte der Holzfeuchte und Rohdichte, Eigenschaften ermittelt nach DIN EN 408

Table 6 Moisture content and density statistics, properties determined according to DIN EN 408

\begin{tabular}{lccccc}
\hline \multirow{2}{*}{ Herkunft } & & \multicolumn{2}{c}{ Holzfeuchte [\%] } & \multicolumn{2}{c}{ Rohdichte $\left[\mathrm{kg} / \mathrm{m}^{3}\right]$} \\
Spessart & $n^{\mathrm{a}}$ & $62\left(=2 \cdot 31^{\mathrm{b}}\right)$ & $176\left(=2 \cdot 88^{\mathrm{b}}\right)$ & 62 & 176 \\
& $\bar{x}$ & 11,0 & 9,64 & 684 & 681 \\
& $\mathrm{~s}$ & 0,681 & 0,822 & 47,7 & 33,8 \\
Nordhessen & $\mathrm{n}^{\mathrm{a}}$ & $112\left(=2 \cdot 56^{\mathrm{b}}\right)$ & $166\left(=2 \cdot 83^{\mathrm{b}}\right)$ & 112 & 166 \\
& $\bar{x}$ & 9,85 & 8,88 & 695 & 672 \\
& $\mathrm{~s}$ & 0,579 & 0,345 & 44,1 & 39,2 \\
Schönbuch & $\mathrm{n}^{\mathrm{a}}$ & $42\left(=2 \cdot 21^{\mathrm{b}}\right)$ & $176\left(=2 \cdot 88^{\mathrm{b}}\right)$ & 42 & 176 \\
& $\bar{x}$ & 10,1 & 9,84 & 687 & 681 \\
& $\mathrm{~s}$ & 0,519 & 0,432 & 34,6 & 32,3 \\
\hline
\end{tabular}

${ }^{\mathrm{a}}$ zwei Brettenden je Prüfkörper

b Stichprobenumfang aus Tabelle 3
In Tabelle 6 sind die statistischen Kennwerte dieser beiden Eigenschaften zusammengestellt. Der Mittelwert der Holzfeuchte bzw. der Rohdichte schwankt bei den sechs Stichproben, die nach Holzherkunft und Sortierverfahren unterschieden werden, zwischen $8,88 \%$ und $11,0 \%$ bzw. $672 \mathrm{~kg} / \mathrm{m}^{3}$ und $695 \mathrm{~kg} / \mathrm{m}^{3}$. Im Hinblick auf das Verkleben der Zinken wurde bei den Prüfkörpern aus visuell sortierten Brettern Herkunft Spessart zwischen zwei beliebigen Brettenden eine Holzfeuchte-Differenz von 5,25\% gemessen. Bei den Brettern aus Nordhessen und dem Schönbuch betrug diese Differenz höchstens 3\%. Damit wird der in DIN EN 386 geforderte Grenzwert von 4\% im Wesentlichen eingehalten. Insofern wurde die technische Trocknung des Buchenholzes bei allen Produzenten zuverlässig durchgeführt. Da die Holzfeuchte der Proben des maschinell sortierten Materials später bestimmt wurde, können damit Unterschiede der Holzfeuchte bis zu 1,4\% zwischen den Proben der visuell und maschinell sortierten Bretter erklärt werden.

\subsection{Biegefestigkeit und dynamischer \\ Biege-Elastizitätsmodul \\ 3.3.1 Visuelle Sortierung}

Abbildung 4 zeigt die Biegefestigkeit der Keilzinkenverbindungen in Abhängigkeit vom dynamischen Biege- 

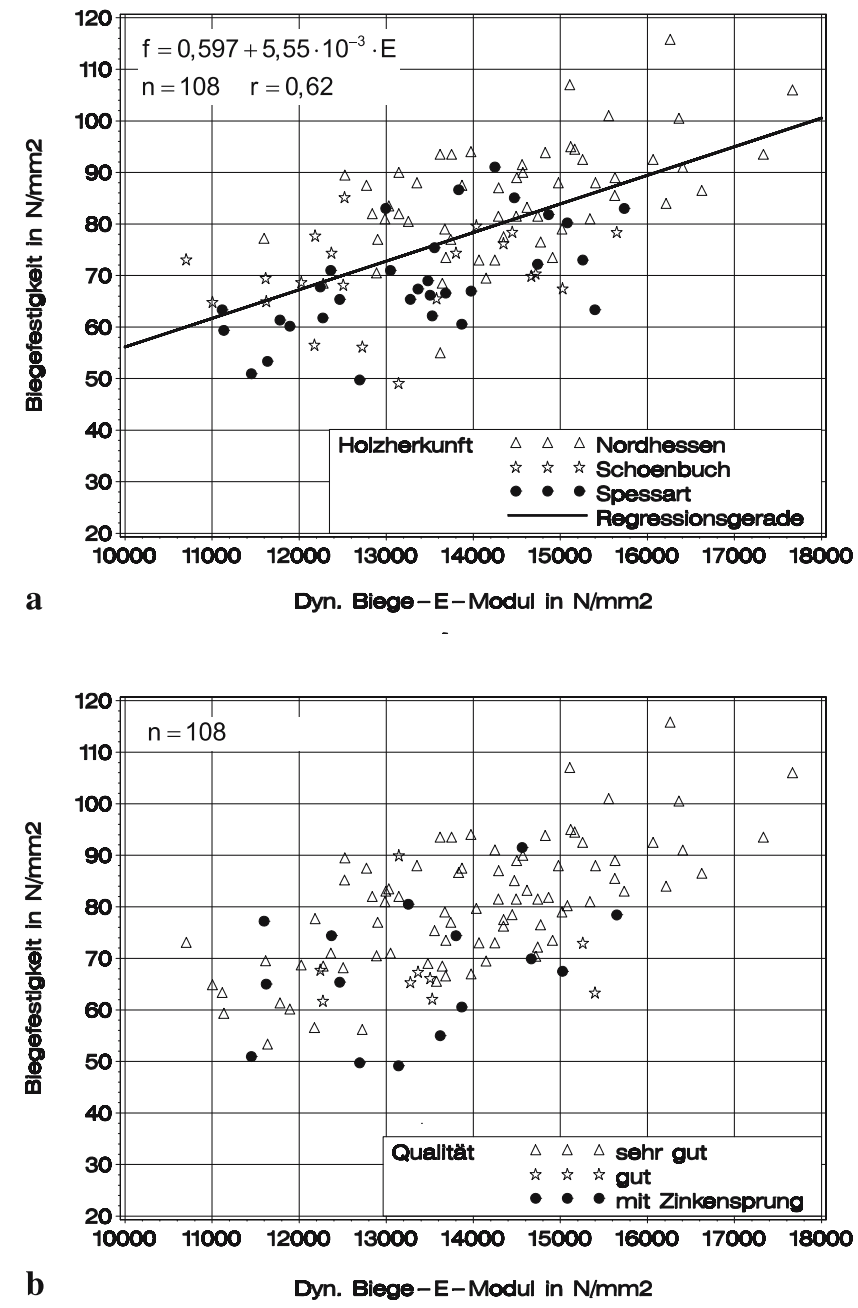

Abb. 4 Keilzinkenbiegefestigkeit in Abhängigkeit vom dynamischen Biege-Elastizitätsmodul; Unterscheidung nach Herkunft (a) und Qualität (b)

Fig. 4 Finger joint bending strength depending on the flexural mode MOE; source differentiation (a) and quality differentiation (b)

Elastizitätsmodul. In Abb. 4a ist die unterschiedliche Herkunft der Bretter durch Symbole gekennzeichnet. Diese kennzeichnen auch drei zeitlich versetzte Produktionstermine, weil während einer Produktion nur Bretter einer Herkunft verarbeitet wurden. Die Regressionsgerade für alle Beobachtungen belegt die Zunahme der Festigkeit mit steigendem dynamischem Biege-Elastizitätsmodul. In Abb. 4b wird der Einfluss der Keilzinkenqualität auf die Festigkeit verdeutlicht. Bei unzureichenden Verklebungen und Zinkensprüngen werden Biegefestigkeiten über $80 \mathrm{~N} / \mathrm{mm}^{2}$ selten beobachtet. Die Prüfkörper mit den drei kleinsten Festigkeitswerten weisen Zinkensprünge auf. In Tabelle 7 sind die statistischen Kennwerte der Biegefestigkeit zusammengestellt. Für die charakteristische Biegefestigkeit können aus Abb. 5 der verteilungsfrei ermittelte Wert von $55,5 \mathrm{~N} / \mathrm{mm}^{2}$ und aus der angepassten log-
Tabelle 7 Statistische Kennwerte der Biegefestigkeit in $\mathrm{N} / \mathrm{mm}^{2}$; visuelle Sortierung; 108 Proben getrennt nach Herkunft

Table 7 Bending strength statistics in $\mathrm{N} / \mathrm{mm}^{2}$; visual strength grading; 108 specimens

\begin{tabular}{lcccc}
\hline & Spessart & Nordhessen & Schönbuch & Alle Proben \\
\hline $\mathrm{n}$ & 31 & 56 & 21 & 108 \\
$\bar{x}$ & 68,8 & 85,2 & 70,0 & 77,5 \\
$\mathrm{~s}$ & 10,4 & 10,6 & 8,7 & 12,9 \\
$5 \%$ & 50,4 & 68,5 & 49,5 & 55,5 \\
$\mathrm{VG}_{95}^{1}$ & $44,5-56,4$ & $62,6-71,5$ & $47,9-60,4$ & $52,1-59,7$ \\
\hline
\end{tabular}

$195 \%$-Vertrauensgrenzen für das 5\%-Quantil ermittelt unter der Annahme normalverteilter Daten

Normalverteilung $57,8 \mathrm{~N} / \mathrm{mm}^{2}$ entnommen werden. Unter Annahme normal verteilter Daten liegen für alle Proben die 95\%-Vertrauensgrenzen für das 5\%-Quantil bei $52,1 \mathrm{~N} / \mathrm{mm}^{2}$ bzw. $59,7 \mathrm{~N} / \mathrm{mm}^{2}$. Eine einfache Varianzanalyse zeigt, dass der Mittelwert der Biegefestigkeit bei der Stichprobe Nordhessen signifikant höher ist als die Mittelwerte der zwei anderen Proben-Kollektive. Das wird durch bessere Holzeigenschaften erklärt: Die Symbole der Holzherkunft Nordhessen befinden sich im Gegensatz zu den anderen eher im rechten Teil des Diagramms in Abb. 4a. Dementsprechend weisen sie auch einen im Mittel höheren dynamischen Biege-Elastizitätsmodul auf. Die gemeinsame Anzahl der Proben aus dem Spessart und Schönbuch, die ein vergleichbares Festigkeitsniveau besitzen, entspricht etwa dem Umfang der Proben aus Nordhessen. Insofern ist die Vereinigung der drei Proben zulässig, weil sowohl niedrige als auch hohe Festigkeitswerte zu etwa gleichen Anteilen repräsentiert werden. Die Festigkeitsunterschiede bei den Mittelwerten von $16,4 \mathrm{~N} / \mathrm{mm}^{2}$ bzw. $15,2 \mathrm{~N} / \mathrm{mm}^{2}$ zwischen den Proben aus Nordhessen und dem Spessart bzw. Schönbuch deuten auf uneinheitliche Festigkeitswerte bei der Grundgesamtheit von Keilzinken aus visuell sortierten Brettern hin.

\subsubsection{Maschinelle Sortierung}

Abbildung 6 zeigt die Beziehung zwischen der Biegefestigkeit und dem dynamischen Biege-Elastizitätsmodul der Proben aus maschinell sortierten Brettern. Weil der dynamische Biege-Elastizitätsmodul der Keilzinkenproben positiv mit dem dynamischen Elastizitätsmodul der Bretter korreliert ist, wird die Klassifizierung der Bretter nach Tabelle 2 in Abb. 6 deutlich. Proben aus Brettern der Klasse 2 liegen im linken und solche der Klasse 5 im rechten Bereich des Diagramms. Eingezeichnet ist eine quadratische Regressionskurve mit 90\%-Vertrauensgrenzen, die mit allen Beobachtungen ermittelt wurde. Diese zeigt, dass die Keilzinkenbiegefestigkeit trotz höherer Anforderungen an den dynamischen Elastizitätsmodul der Bretter 
Abb. 5 Anpassung einer log-Normalverteilung an die empirischen Daten;

Keilzinkenproben aus visuell sortierten Brettern

Fig. 5 Empirical data and fitted lognormal distribution; specimens manufactured from visually strength graded boards
Abb. 6 Biegefestigkeit in Abhängigkeit vom dynamischen Biege-Elastizitätsmodul; quadratische Regressionskurve und $90 \%$-Vertrauensgrenzen Fig. 6 Bending strength depending on the flexural mode MOE; quadratic regression curve and $90 \%$ confidence limits
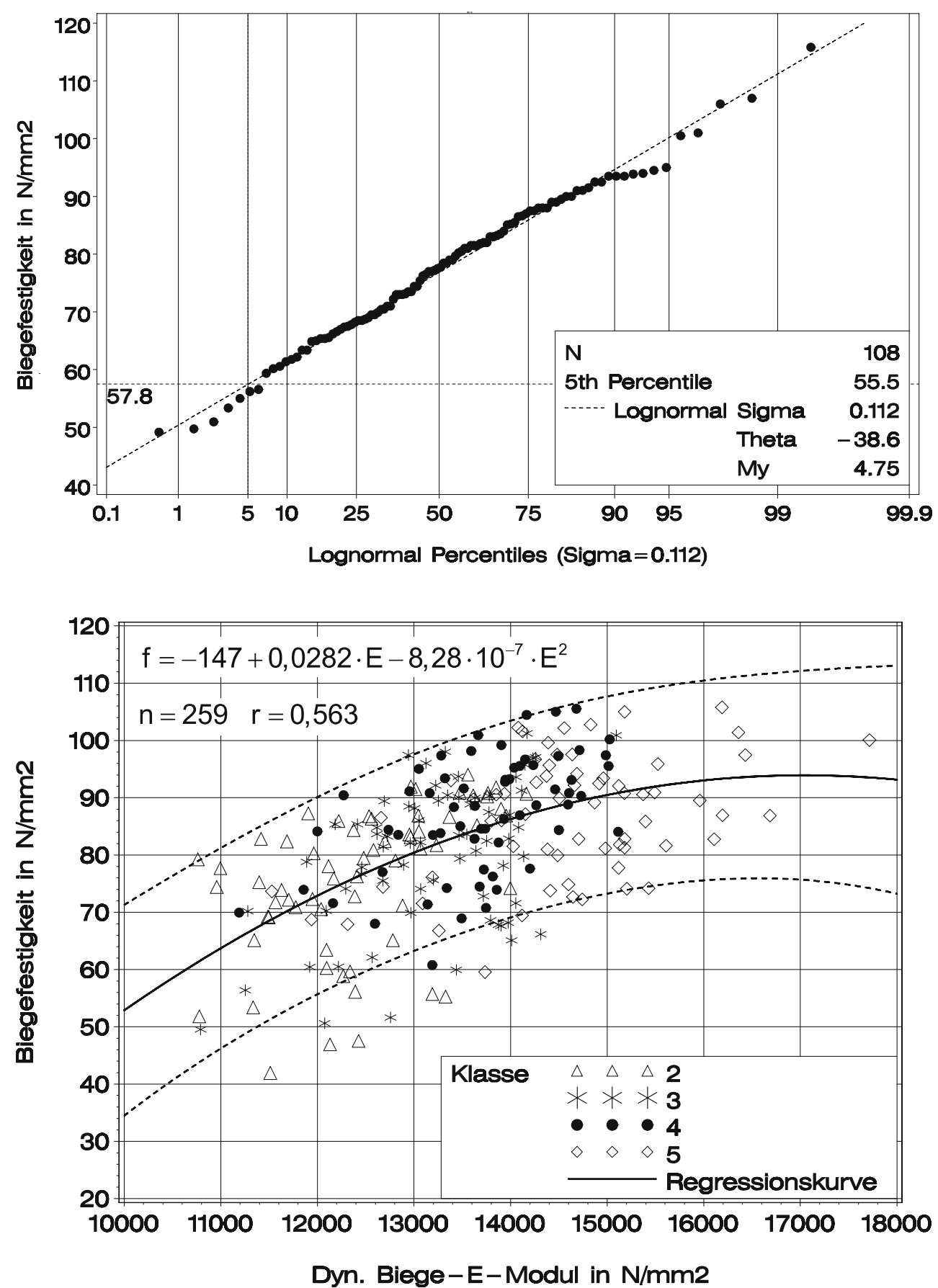

ab einem gewissen Niveau nicht weiter ansteigt. Diese Tendenz wird in Abb. 7 verdeutlicht. Für jede Herkunft sind der Mittelwert und das 5\%-Quantil der Keilzinkenbiegefestigkeit über der Klasse der keilgezinkten Bretter aufgetragen. Für beide Kennwerte zeigt sich, dass ab Klasse 4 keine nennenswerte Zunahme der Festigkeit bei den untersuchten Proben nachgewiesen werden kann. Abbildung 8 hingegen zeigt, dass der mittlere dynamische Biege-Elastizitätsmodul der Proben zwischen den Klassen 4 und 5 am stärksten ansteigt. Unter Beachtung der beim
Holz sonst engen positiven Korrelation zwischen Elastizitätsmodul und Festigkeit erscheint dies als Phänomen. Eine Erklärung dafür könnte sein, dass mit zunehmender Holzqualität beim Biegebruch der hier untersuchten Proben häufiger Versagensformen auftreten, die auch von der Verklebung zwischen den Zinkenflanken (z.B. Herausziehen der Zinken) und nicht nur von der Holzqualität (z.B. Holzversagen im Zinkengrund) abhängig sind. Eine Überprüfung dieser beiden Versagensformen gab dafür einige Hinweise. 
Abb. 7 Mittelwert (oben) und 5\%-Quantil (unten) der Biegefestigkeit in Abhängigkeit von der Klasse der verzinkten Bretter

Fig. 7 Mean (top) and 5th percentile (bottom) bending strength value as a function of grade of connected boards

\section{Abb. 8 Mittelwert des} dynamischen

Biege-Elastizitätsmoduls in Abhängigkeit von der Klasse der verzinkten Bretter

Fig. 8 Mean value of the flexural mode MOE as a function of grade of connected boards
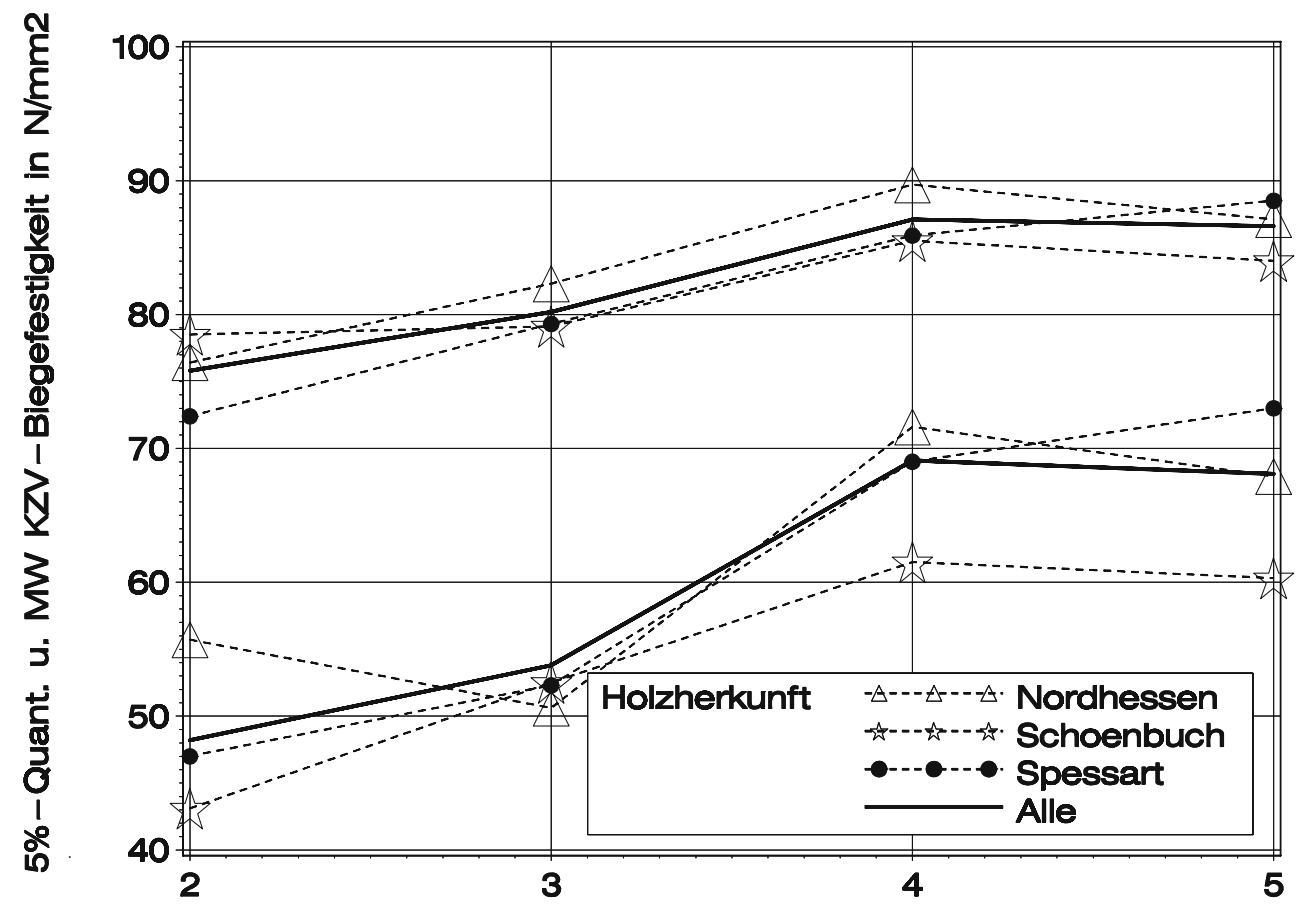

Klasse der verzinkten Bretter

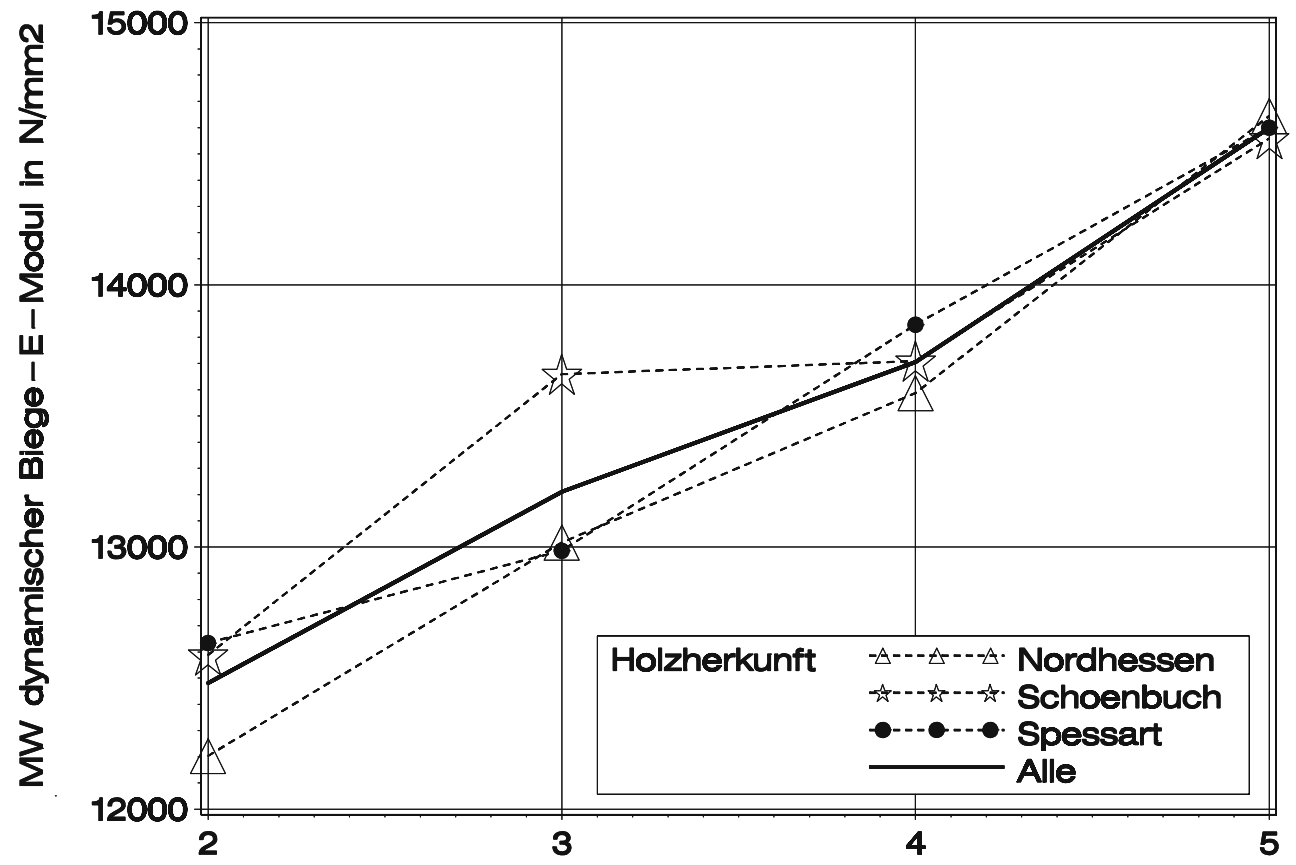

Klasse der verzinkten Bretter
Bemerkenswert ist, dass eine maschinelle Sortierung in die Klassen 2 und 3 eine charakteristische Biegefestigkeit von $48,2 \mathrm{~N} / \mathrm{mm}^{2}$ bzw. 53,8 N/mm² zur Folge hat (Tabelle 8). Der ermittelte charakteristische Wert für die Klasse 3 liegt im unteren Bereich des 95\%-Vertrauensintervalls der charakteristischen Keilzinkenbiegefestigkeit für eine visu- elle Sortierung in die Klassen LS10 und LS13. Deshalb beginnt der praktische Nutzen einer maschinellen Sortierung für Bretter mit einem dynamischen Elastizitätsmodul erst oberhalb von $15000 \mathrm{~N} / \mathrm{mm}^{2}$ in den Klassen 4 oder 5 . Dann findet bezüglich der charakteristischen Festigkeit sowohl das Holz als auch die Keilzinkenverbindung betref- 
Tabelle 8 Statistische Kennwerte der Biegefestigkeit in $\mathrm{N} / \mathrm{mm}^{2}$; maschinelle Sortierung; 259

Proben getrennt nach Herkunft und Klasse

Table 8 Bending strength statistics in $\mathrm{N} / \mathrm{mm}^{2}$; mechanical strength grading; 259 specimens

\begin{tabular}{lcccccc}
\hline & & \multicolumn{5}{c}{ Klasse } \\
Herkunft & \multicolumn{1}{c}{3} & 3 & 4 & 5 & $4+5^{1}$ \\
\hline Spessart & $\mathrm{n}$ & 21 & 25 & 18 & 24 & 42 \\
& $\bar{x}$ & 72,4 & 79,3 & 85,9 & 88,5 & 87,4 \\
& $\mathrm{~s}$ & 14,7 & 12,5 & 9,42 & 9,15 & 9,24 \\
Nordhessen & $5 \%$ & 47 & 52,3 & 69,0 & 73,0 & 71,0 \\
& $\mathrm{n}$ & 20 & 22 & 22 & 19 & 41 \\
& $\bar{x}$ & 76,4 & 82,3 & 89,7 & 87,1 & 88,5 \\
Schönbuch & $\mathrm{s}$ & 9,73 & 13,2 & 10,1 & 10,6 & 10,3 \\
& $5 \%$ & 55,7 & 51,2 & 71,6 & 67,9 & 71,4 \\
& $\mathrm{n}$ & 22 & 22 & 22 & 22 & 44 \\
Alle & $\bar{x}$ & 78,5 & 79,1 & 85,5 & 84,0 & 84,7 \\
Wuchsgebiete & $\mathrm{s}$ & 13,3 & 12,2 & 11 & 12,3 & 11,6 \\
& $5 \%$ & 43,1 & 52,5 & 61,5 & 60,3 & 62 \\
& $\mathrm{n}$ & 63 & 69 & 62 & 65 & 127 \\
& $\bar{x}$ & 75,8 & 80,2 & 87,1 & 86,6 & 86,8 \\
& $\mathrm{~s}$ & 12,9 & 12,5 & 10,2 & 10,8 & 10,5 \\
& $5 \%$ & 48,2 & 53,8 & 69,1 & 68,1 & 68,8 \\
& $\mathrm{VG}_{95}^{2}$ & $48,9-58,9$ & $54,4-63,7$ & $65,7-73,7$ & $64,2-72,4$ & $66,5-72,2$ \\
\hline
\end{tabular}

${ }^{1}$ Vereinigung der Klassen 4 und 5

2 95\%-Vertrauensgrenzen für das 5\%-Quantil ermittelt unter der Annahme normalverteilter Daten
Abb. 9 Anpassung einer log-Normalverteilung an die empirischen Daten;

Keilzinkenproben aus Brettern der Klassen 4 und 5

Fig. 9 Empirical data and fitted lognormal distribution; finger joint specimens manufactured from mechanically strength graded boards of grades 4 and 5

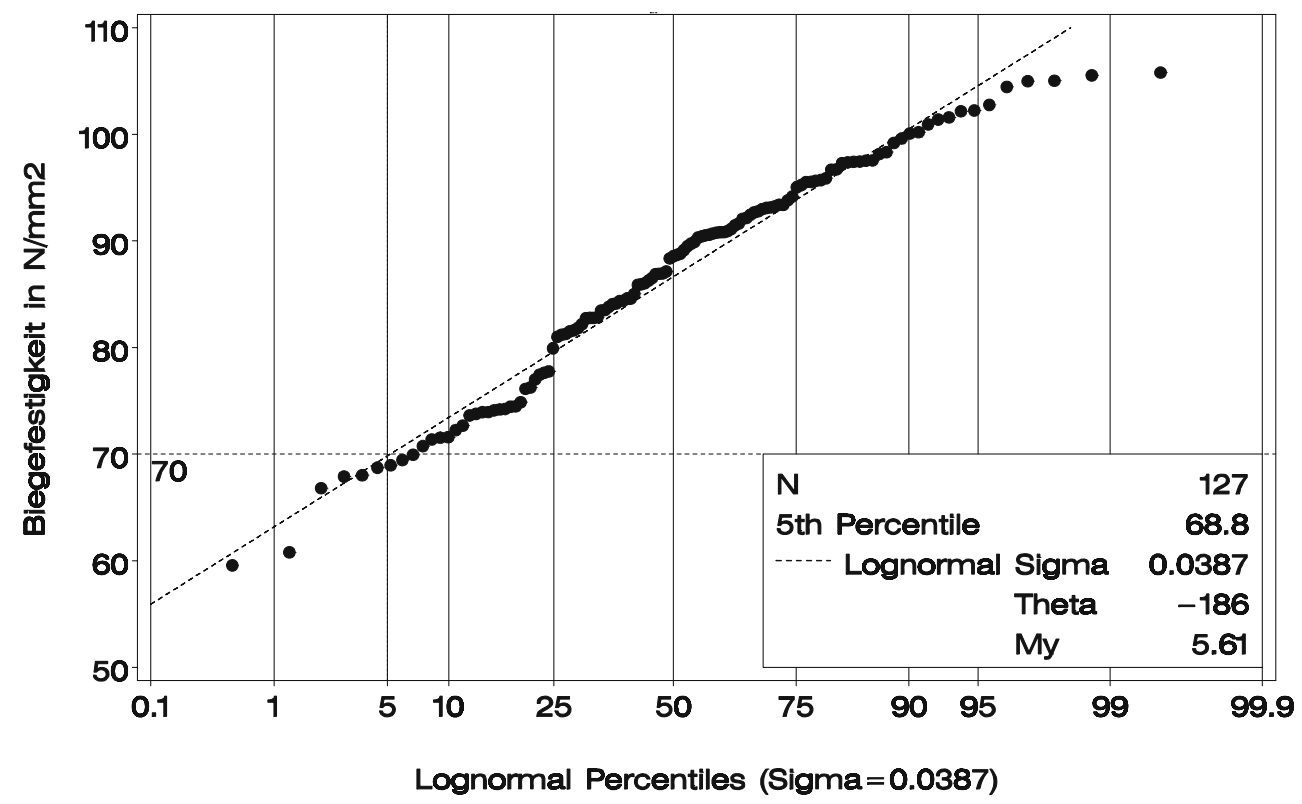

fend - eine klare Abgrenzung zur visuellen Sortierung statt (siehe hierzu auch Tabelle 1). In Übereinstimmung mit den Sortiermodellen MSa und MSb, für die Bretter mit einem dynamischen Elastizitätsmodul $>15000 \mathrm{~N} / \mathrm{mm}^{2}$ erforderlich sind, enthält Tabelle 8 in der letzten Spalte auch eine gemeinsame Auswertung für die Klassen 4 und 5. Dementsprechend ist für die 127 Proben dieser beiden Klassen in Abb. 9 die empirische Verteilung und die angepasste logNormalverteilung der Biegefestigkeit dargestellt. Die Gegenüberstellung zeigt eine verteilungsfrei ermittelte charakteristische Keilzinkenbiegefestigkeit von $68,8 \mathrm{~N} / \mathrm{mm}^{2}$ und für die theoretische Verteilung einen Wert von $70 \mathrm{~N} / \mathrm{mm}^{2}$. Bei Betrachtung der Stichproben Spessart und Nordhes- sen für die Klassen 4 und 5 werden sogar charakteristische Biegefestigkeiten über $70 \mathrm{~N} / \mathrm{mm}^{2}$ erreicht. Die $95 \%$ Vertrauensgrenzen für das 5\%-Quantil der 127 Proben liegen unter der Annahme normalverteilter Daten zwischen $66,5 \mathrm{~N} / \mathrm{mm}^{2}$ und $72,2 \mathrm{~N} / \mathrm{mm}^{2}$. Die einfache Varianzanalyse zeigt, dass innerhalb einer Klasse die Mittelwerte der Keilzinkenbiegefestigkeit je Herkunft nicht signifikant voneinander abweichen. Insofern begünstigt die maschinelle Sortierung im Vergleich zur visuellen Sortierung homogene Festigkeiten bei der Grundgesamtheit der Keilzinken aus maschinell sortierten Brettern. Entsprechend gering sind auch die Abweichungen beim Mittelwert zwischen den gestrichelten Linien und der durchgezogenen Linie in Abb. 7. 


\subsection{Festigkeitswerte von nicht ordnungsgemäßen Keilzinkenverbindungen}

Um zu zeigen, dass dem einwandfreien Zustand der Brettenden hinsichtlich der Keilzinkenbiegefestigkeit eine große Bedeutung zukommt, werden in diesem Abschnitt die Festigkeitswerte nicht ordnungsgemäßer Keilzinkenproben dargestellt. Sie blieben in den Auswertungen der Abschn. 3.3.1 und 3.3.2 unberücksichtigt. Es handelt sich dabei um 39 Prüfkörper, bei denen Äste, Risse, festigkeitsmindernde Fäule oder Ähnliches im unzulässigen Abstand zum Zinkengrund beobachtet wurden.

Bei 31 Proben aus visuell sortierten Brettern beträgt der Mittelwert der Biegefestigkeit nach Abb. 10a nur $59,8 \mathrm{~N} / \mathrm{mm}^{2}$. Das entspricht nur 77\% des Mittelwertes der 108 ordnungsgemäßen Proben. Das 5\%-Quantil liegt bei $28,1 \mathrm{~N} / \mathrm{mm}^{2}$ und ist damit nur halb so groß. Zahlreiche Bretter des Ausgangsmaterials ließen nur an einem Brettende eine ordnungsgemäße Keilzinkenverbindung zu. Insofern hatte die kontinuierliche Herstellung „Brett an Brett“ die vergleichsweise hohe Anzahl von 31 Proben zur Folge.

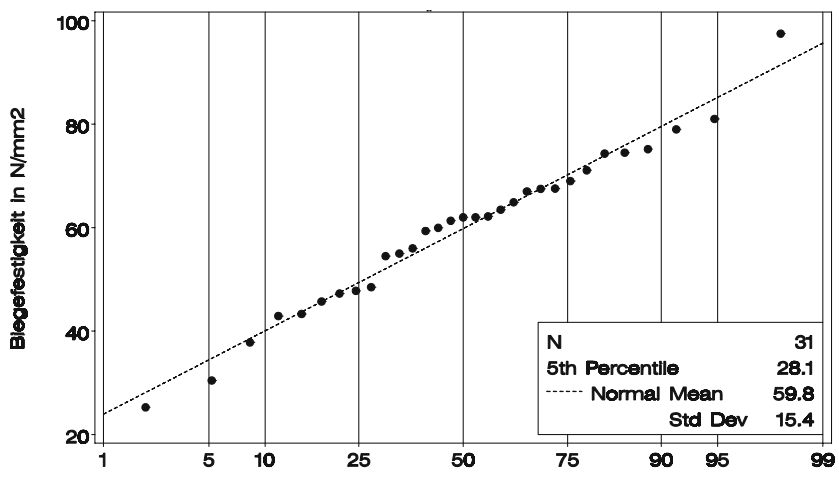

a

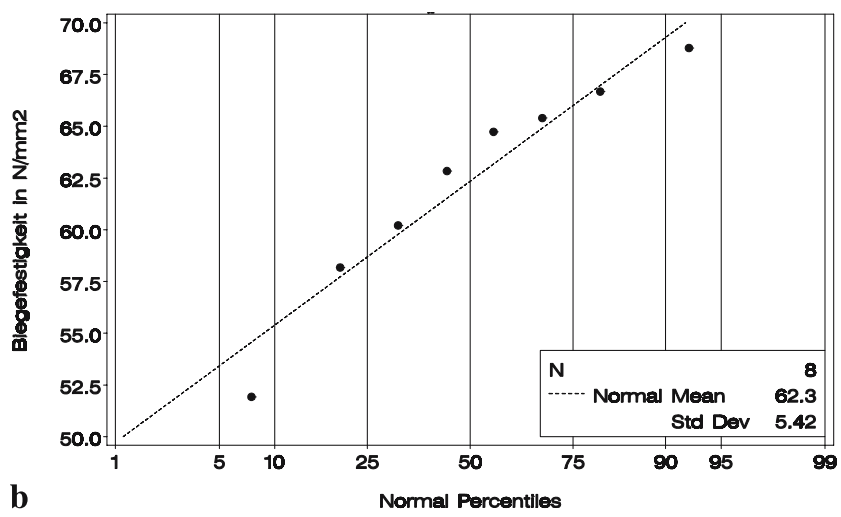

Abb. 10 Verteilung der Biegefestigkeit von Keilzinken, die nicht ordnungsgemäß ausgeführt wurden; Proben aus visuell sortierten Brettern (a) und Proben aus maschinell sortierten Brettern (b)

Fig. 10 Bending strength distribution of rejects; specimens from visually strength graded boards (a) and from mechanically strength graded boards $(\mathbf{b})$
8 Proben aus maschinell sortierten Brettern der Klassen 4 und 5 weisen nach Abb. 10b eine mittlere Biegefestigkeit von nur $62,3 \mathrm{~N} / \mathrm{mm}^{2}$ auf. Das entspricht nur $72 \%$ des Mittelwertes der ordnungsgemäßen Proben. Diese 8 Prüfkörper wurden genauer untersucht, weil ihre Festigkeitswerte den geschätzten charakteristischen Wert von $70 \mathrm{~N} / \mathrm{mm}^{2}$ für maschinell sortierte Bretter empfindlich stören würden. Für die Einstufung in Qualität 4 waren die folgenden Merkmale in unmittelbarer Nähe zu den Zinken ausschlaggebend. Bei einer Probe lag ein Ast vor. Abbildung 11a zeigt dazu den Einfluss des Astes auf das Bruchbild. Einer weiteren konnte nach Schmidt (1993) Weißfäule mit schwarzen Demarkationslinien zugeordnet werden. Diesen Prüfkörper zeigt Abb. 11b. In den übrigen 6 Fällen konnte am Brettende mittels Gutachten ${ }^{1}$ Moderfäule nachgewiesen werden, die im gegenwärtigen Stadium die Festigkeit der Zellwände bereits geschwächt hat. Abbildung 11c zeigt im Tangentialschnitt die Zellen eines Holzstrahls, dessen Sekundärwände bereits geschädigt sind. Der Pilz „Paecilomyces variotii“ hat mit großer Wahrscheinlichkeit die Moderfäule verursacht. Da der nachgewiesene Moderfäulepilz nasses Holz befällt, kommt ein Befall des frisch geschlagenen Stammholzes oder während feuchter Lagerung in Frage. Die Proben mit Moderfäule waren nur der Herkunft Nordhessen zuzuordnen.

In Anbetracht des ungünstigen Einflusses von unzulässigen Merkmalen im Bereich der Zinkung auf deren Festigkeit ist eine gründliche Begutachtung und Vorbereitung der Brettenden vor der Herstellung der Zinken erforderlich. Erst dadurch werden die charakteristischen Keilzinkenbiegefestigkeiten, die hier für visuell und maschinell sortierte Buche nachgewiesen wurden, auch wirklich sichergestellt.

\section{Zusammenfassung}

Der Einfluss einer visuellen oder maschinellen Sortierung von Buchenbrettern auf die Biegefestigkeit von Keilzinkenverbindungen wurde aufgezeigt. Es konnte nachgewiesen werden, dass eine visuelle Sortierung von Buchenbrettern in die Sortierklassen LS10 oder LS13 eine charakteristische Keilzinkenbiegefestigkeit von $56 \mathrm{~N} / \mathrm{mm}^{2}$ ermöglicht. Dieser Wert wurde an 108 Proben ermittelt. Signifikante Unterschiede der Festigkeit bezüglich der Sortierklassen LS10 und LS13 sind aufgrund der gleichen Rohdichte am Brettende in den beiden Sortierklassen nicht zu erwarten. Eine maschinelle Sortierung nach dem dynamischen Elastizitätsmodul, ermittelt aus Längsschwingungen, führt erst oberhalb eines Grenzwertes von $15000 \mathrm{~N} / \mathrm{mm}^{2}$ zu nennenswert höheren charakteristischen Biegefestigkeiten der Keilzinken als bei einer visuellen Sortierung. 127 Keilzinken-

\footnotetext{
${ }^{1}$ An dieser Stelle wird Herrn Dr. rer. nat. K. Grimm, Karlsruhe für das Gutachten gedankt.
} 
Abb. 11 Ast (a), Weißfäule (b) und Tangentialschnitt $400 \times$, Besiedelung des Holzes über Holzstrahlen, Holzstrahlzelle (Pfeil) mit teilweise abgebauter Sekundärwand, Aufnahme K. Grimm (c)

Fig. 11 Knot (a), decay (b) and tangential cross section $400 \times$, parenchyma cell (arrow) showing a decayed cell wall, photo by K. Grimm (c)
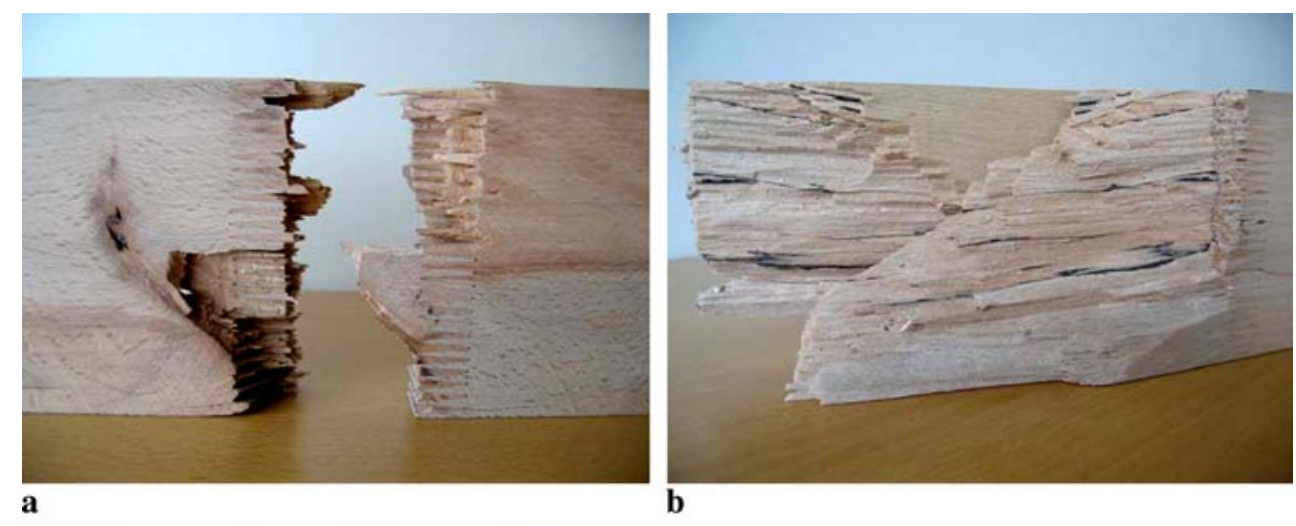

c

proben aus solchen Brettern weisen eine charakteristische Biegefestigkeit von $70 \mathrm{~N} / \mathrm{mm}^{2}$ auf. Alle untersuchten Proben wurden mit dem Profil 15/3,8 und mit einem MelaminKleber an drei unterschiedlichen Produktionsterminen bei einem Brettschichtholzproduzenten hergestellt. Es konnte gezeigt werden, dass die Kriterien bezüglich der Brettenden nach DIN EN 385 auch bei Keilzinken aus Buche große Bedeutung haben. An 39 Proben wurde festgestellt, dass Äste, Risse, Mark und Fäule im Bereich der Zinkung die Festigkeit im Mittel um bis zu 28\% reduzieren.

Mit den Ergebnissen dieser Arbeit kann die Herstellung von GL28 bis GL36 aus visuell sortierten Buchenbrettern und GL40 bis GL48 aus maschinell sortierten Brettern nun abschließend geregelt werden.

\section{Literatur}

Blaß HJ, Denzler JK, Frese M, Glos P, Linsenmann P (2004) Biegefestigkeit von Brettschichtholz aus Buche (Band 1 Karlsruher
Berichte zum Ingenieurholzbau). Universitätsverlag Karlsruhe, Karlsruhe

DIN EN 385, Ausgabe März 2002. Keilzinkenverbindungen im Bauholz - Leistungsanforderungen und Mindestanforderungen an die Herstellung

DIN EN 386, Ausgabe April 2002. Brettschichtholz - Leistungsanforderungen und Mindestanforderungen an die Herstellung

DIN EN 408, Ausgabe April 1996. Bauholz für tragende Zwecke und Brettschichtholz - Bestimmung einiger physikalischer und mechanischer Eigenschaften

DIN 1052, Ausgabe August 2004. Entwurf, Berechnung und Bemessung von Holzbauwerken - Allgemeine Bemessungsregeln und Bemessungsregeln für den Hochbau

DIN 4074 Teil 5, Ausgabe Juni 2003. Sortierung von Holz nach der Tragfähigkeit, Laubschnittholz

Ehlbeck J, Colling F, Görlacher R (1984) Einfluss keilgezinkter Lamellen auf die Biegefestigkeit von Brettschichtholzträgern. Forschungsbericht der Versuchsanstalt für Stahl, Holz und Steine, Abteilung Ingenieurholzbau, Karlsruhe, Universität (TH)

Görlacher R (1984) Ein neues Messverfahren zur Bestimmung des Elastizitätsmoduls von Holz. Holz Roh- Werkst 42:219-222

Schmidt O (1993) Holz- und Baumpilze. Springer, Berlin Heidelberg New York 